\title{
HREM Study of Fullerenes Impact in a Metallic Matrix
}

\author{
J. Pacaud*, A. Michel*, C. Jaouen*, F. Pailloux*, S. DellaNegra** \\ * Laboratoire de Métallurgie Physique, UMR 6630, SP2MI, Ave P. et M. Curie, 86962 Chasseneuil, \\ France \\ ** Institut de Physique Nucléaire, CNRS-IN2P3, F-91406 Orsay Cedex, France
}

In the field of High Resolution Electron Microscopy, there is an increasing need to extract data that can be compared with models. New developments in image processing allows to extract quantitative information from HREM micrographs on a very local scale and thus gives access to extremely localized structural analysis.

In this study we used Fourier filtering and geometrical phase shift reconstruction to reach structural properties of $\mathrm{FeAl}$ crystal in and in the vicinity of an impact of $30 \mathrm{MeV} \mathrm{C} \mathrm{C}_{60}$ cluster. Due to the size of the object the HRTEM coupled with image processing appears the only tool able to give access to this information.

Irradiation with $\mathrm{C}_{60}$ clusters with an energy of about $30 \mathrm{MeV}$ induce a very high level of electronic excitation transferred to the crystal lattice through a mechanism of electron-phonon coupling that is still unclear. Experimental evidences for local heating, transition to liquid state, Coulombian explosion or shockwave propagation are difficult to obtain due to the scale of the phenomenon. $\mathrm{FeAl}(40 \%$ atomic) alloys crystallise in a $\mathrm{B} 2$ structure. Both atomic species are located on 2 simple cubic sub-lattices translated with respect to each other by a $1 / 2\langle 111\rangle$ vector. From low temperature up to almost the melting point, it shows a long distance order even after rapid quench from liquid state. Morphology of the impact after irradiation may enlighten the mechanisms of damaging for this high electronic energy deposition.

Irradiations were performed at the TANDEM accelerator in Orsay with $30 \mathrm{MeV} \mathrm{C}_{60}$ clusters. [100], [110] and [111] orientations were used to check an effect of crystalline anisotropy. HREM was performed on a JEOL 3010 operating at $300 \mathrm{keV}$ with an ARP pole piece giving a point resolution of $0.19 \mathrm{~nm}$.

Figure 1 shows an impact in a [110] sample. The size of the impact is about $20 \mathrm{~nm}$. This is much larger than the tube where the carbon atoms travel. The impact is limited by (111) planes. The numerical diffractograms inside and outside the track clearly shows the disappearance of the ordering spot inside the impact. It means that this region is chemically disordered and thus may have suffered a melting followed by an extremely rapid quenching.

In Figure 2, on the left, the impact in a [100] oriented sample has (110) limiting planes. The core is still chemically disordered. On the right, the geometrical phase shift reconstruction using a [110] reference shows that the deformation of the lattice is confined in the $\langle 110\rangle$ direction and the disordering of the core of the track induce an elastic displacement field up to $30 \mathrm{~nm}$ from the impact.

Authors gratefully acknowledge the TANDEM in Orsay for irradiation time. 


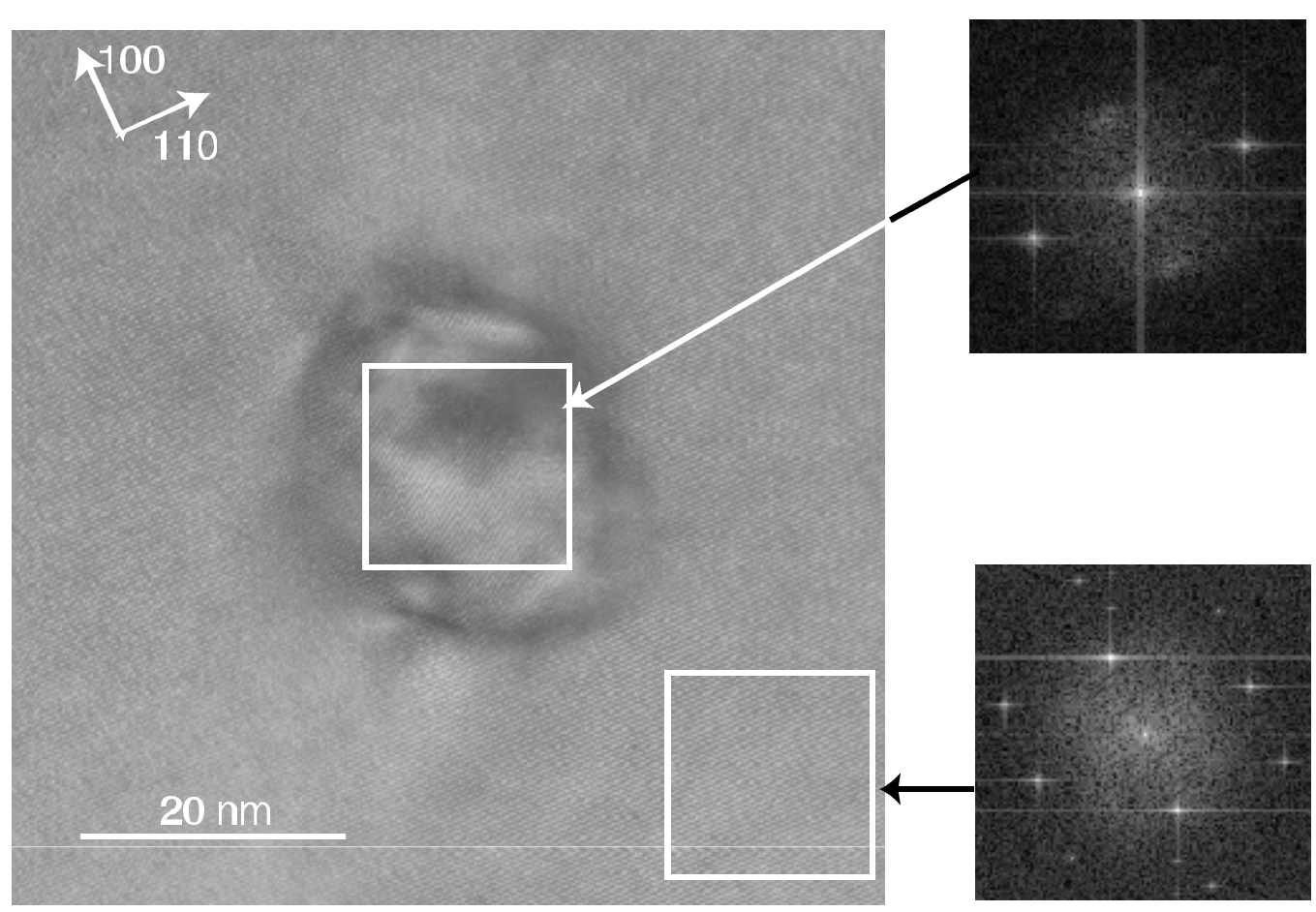

FIG 1: Impact of $\mathrm{a} \mathrm{C}_{60}$ in a [110] oriented FeAl matrix. Impact is clearly limited by (111) planes. Fourier Analysis of different part of the image shows that the core of the impact is chemically disordered.
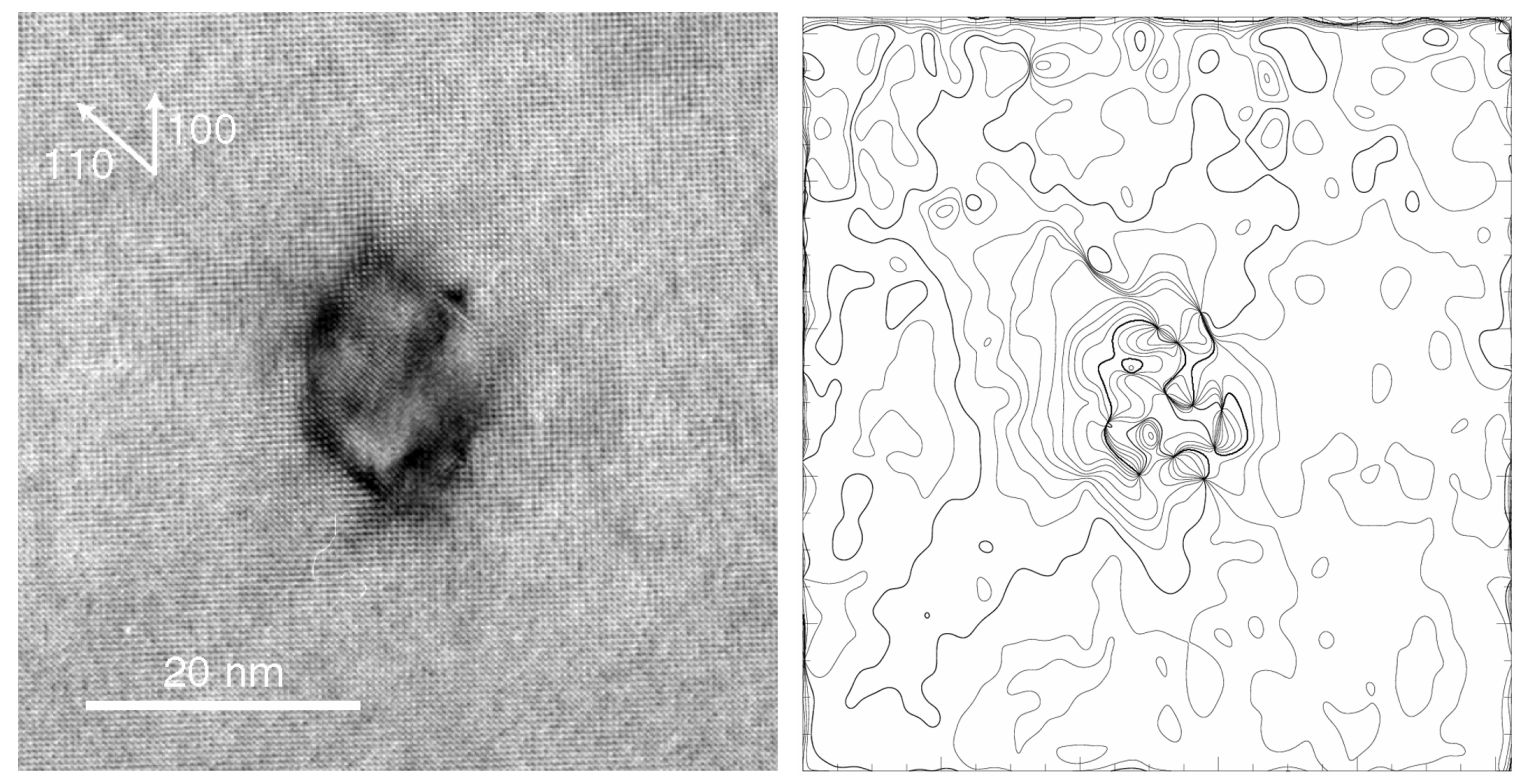

FIG 2 : On the left, HREM micrograph of an impact of $\mathrm{C}_{60}$ in a [100] oriented sample. Core is also chemically disordered. On the right, the geometrical phase shift reconstruction shows strains in the surrounding crystal along $<110>$ directions. 